tious diseases. PloS Pathog. 2006;2:e62.

3. Gruver AL, Hudson LL, Sempowski GD. Immuno-senescence of ageing. J Pathol. 2007;211:144-56.

4. Weiskopf D, Weinberger B, Grubeck-Loebenstein B. The aging of the immune system. Transpl Int.
2009;22:1041-50.

5. Zhou F, Yu T, Du R, et al. Clinical course and risk factors for mortality of adult in patients with COVID-19 in Wuhan, China: A retrospective cohort study. Lancet. 2020;395:1054-62.

\section{Requesting National Guidelines for Screen Time in Indian Children from IAP}

We read with great interest the research article by Meena, et al. [1] on screen time in children by $15-18$ months of age. We wish to share our survey findings of 109 well children (12-36 months) at three hospitals in Bangalore between September, 2016 to April, 2017. We found that the mean (SD) screen time exposure was 120 (96.2) minutes (range 0-540 min) in this age group, similar to the study by Meena, et al. [1]. Television $(69 \%)$, mobile phones (66\%), tablet $(27.5 \%)$ and laptop (22\%) were the most common electronic media used in our study. We found rhymes $(89 \%)$ commercial advertisements (62\%), educational videos $(36 \%)$, and cartoons $(20 \%)$ constituted majority of the activities during screen time. Importantly, $73 \%$ of the parents used screen time to help the child in eating, 51\% for entertain-ment, and $34.8 \%$ as a distraction to give the caregiver some free time. Contrary to their findings, we found that only $7 \%$ of the parents thought screen time was good for the child. We found that $26 \%$ of these children had delay in speech for their respective ages. An association with speech delay and screen time has also been reported earlier [2,3].
We agree with the authors that it is the need of the hour to not only provide Indian guidelines for screen time in toddlers to parents but also to make them aware of possible adverse effects it may cause in their toddler's speech development. We are eagerly waiting for guidelines of Indian Academy of Pediatrics on screen time for children to address this important issue, and to communicate these to parents in addition to practitioners.

Ethics clearance: Institutional Ethics Committee, Kids Clinic India Pvt Ltd; No. ECR/791/ Inst/KA/ 2015/RR-18, dated August 20, 2016.

\section{Meghna Manocha* and MeenaVenkatraman Cloudnine Hospital Old Airport Road, Bangalore, Karnataka, India. *drmeghna@cloudninecare.com}

\section{REFERENCES}

1. Meena P, Gupta P, Shah D. Screen time in Indian children by 15-18 months of age. Indian Pediatr. 2020;57:1033-36.

2. Service I-AN. Smartphones, Tablets May Cause Speech Development Delays in Kids. NDTV Gadgets360Com 2017. Accessed April 20, 2018. Available from: https:// gadgets.ndtv.com/science/news/smartphones-tablets-maycause-speech-development-delays-in-kids-report1689995

3. Anderson DR, Pempek TA. Television and very young children. Am Behav Sci. 2005;48:505-22.

\section{The Need for Geographic Location Specific Optical Density Cut-offs for IgM ELISA Serology to Diagnose Scrub Typhus in Children}

We read with interest the article on detection of immunoglobulin $\mathrm{M}$ and immunoglobulin $\mathrm{G}$ antibodies against Orientia tsutsugamushi for scrub typhus (ST) diagnosis by Gupte, et al. [1]. This study was done to estimate the regional cut-off of optical density (OD) values of serum IgM antibodies by ELISA for the diagnosis of ST. Authors had successfully determined regional cut off of OD value for IgM antibodies, which will be utilized in diagnosing ST in that area. This study has shed light into one of the most obvious pitfalls in diagnosing ST by serology (IgM ELISA) i.e., using an inaccurate, arbitrary OD value cut off for diagnosis. This has serious implications that are applicable beyond the study population.

We would like to raise two pertinent points based on the study conclusions. Most studies conducted in pediatric ST using serology by IgM ELISA used $0.5 \mathrm{OD}$ as an arbitrary cut off in accordance with the definition of 'probable case' by the IAP guidelines on rickettsial diseases in children [2]. Since OD of 0.5 is much lower than most of the cut offs reported from Southeast Asia, where ST is endemic in many parts, all these studies with OD cut off of 0.5 might have actually over diagnosed ST and hence the results have to be cautiously interpreted [3-5]. Since the OD cut off is going to be influenced by geographical location and degree of endemicity, it is imperative to use location specific OD cut off to diagnose ST [4]. Conducting epidemiological studies to identify the OD cut 